\title{
Determination of the Nitrogen Vacancy as a Shallow Compensating Center in GaN Doped with Divalent Metals
}

\author{
J. Buckeridge, ${ }^{1,}$ C. R. A. Catlow, ${ }^{1}$ D. O. Scanlon, ${ }^{1,2}$ T. W. Keal, ${ }^{3}$ P. Sherwood, ${ }^{3}$ M. Miskufova, ${ }^{1}$ \\ A. Walsh, ${ }^{4}$ S. M. Woodley, ${ }^{1}$ and A. A. Sokol ${ }^{1, \dagger}$ \\ ${ }^{1}$ University College London, Kathleen Lonsdale Materials Chemistry, Department of Chemistry, 20 Gordon Street, \\ London WC1H OAJ, United Kingdom \\ ${ }^{2}$ Diamond Light Source Ltd., Diamond House, Harwell Science and Innovation Campus, Didcot, \\ Oxfordshire OX11 ODE, United Kingdom \\ ${ }^{3}$ Scientific Computing Department, STFC, Daresbury Laboratory, Daresbury, Warrington WA4 4AD, United Kingdom \\ ${ }^{4}$ Centre for Sustainable Chemical Technologies and Department of Chemistry, University of Bath, \\ Claverton Down, Bath BA2 7AY, United Kingdom \\ (Received 28 August 2014; published 7 January 2015)
}

\begin{abstract}
We report accurate energetics of defects introduced in $\mathrm{GaN}$ on doping with divalent metals, focusing on the technologically important case of $\mathrm{Mg}$ doping, using a model that takes into consideration both the effect of hole localization and dipolar polarization of the host material, and includes a well-defined reference level. Defect formation and ionization energies show that divalent dopants are counterbalanced in GaN by nitrogen vacancies and not by holes, which explains both the difficulty in achieving $p$-type conductivity in $\mathrm{GaN}$ and the associated major spectroscopic features, including the ubiquitous $3.46 \mathrm{eV}$ photoluminescence line, a characteristic of all lightly divalent-metal-doped GaN materials that has also been shown to occur in pure GaN samples. Our results give a comprehensive explanation for the observed behavior of GaN doped with low concentrations of divalent metals in good agreement with relevant experiment.
\end{abstract}

DOI: 10.1103/PhysRevLett.114.016405

PACS numbers: 71.55.Eq, 61.72.J-, 78.55.Cr

Gallium nitride, a wide-gap semiconductor used for a range of applications, e.g., solid-state lighting, high power microelectronics, is an essential component of commercial blue light emitting diodes, which requires both $n$ - and $p$ type conducting layers [1]. While it has been intensively researched for several decades, many puzzling features still remain. For instance, $\mathrm{GaN}$ is natively $n$ type, indicating the presence of shallow donors, but whether these centers are native point defects (e.g., nitrogen vacancies) or unwanted impurities incorporated during growth (such as hydrogen, oxygen, or silicon) remains a source of controversy $[2,3]$.

Industrially, the production of $p$-type $\mathrm{GaN}$ is still the main bottleneck. The only successful approach has been to dope with $\mathrm{Mg}$, but there are many associated problems: large concentrations of $\mathrm{Mg}$ close to the solubility limit, as well as processing, such as thermal annealing or electron irradiation, are required to achieve significant $p$-type activation; high dislocation densities limit hole mobilities; there is a residual $n$-type concentration that must be overcome; and self-compensation may limit activation [4,5].

At low temperature ( $T$ ), the band gap of $\mathrm{GaN}$ is $3.503 \mathrm{eV}$ [6]. Experimental techniques such as photoconductance, photoluminescence (PL), and deep level transient

Published by the American Physical Society under the terms of the Creative Commons Attribution 3.0 License. Further distribution of this work must maintain attribution to the author(s) and the published article's title, journal citation, and DOI. spectroscopy indicate that the $\mathrm{Mg}_{\mathrm{Ga}}$ acceptor state lies $\sim 0.150-0.265 \mathrm{eV}$ above the valence band maximum (VBM) [7], corroborated by theoretical studies [8,9]. With such a deep level, however, why Mg doping results in $p$-type conductivity is not well understood, although theories have been proposed involving defect complexes and hydrogen impurities [10-12], with some experimental support $[13,14]$. Similar acceptor levels are found for other divalent dopants including $\mathrm{Zn}, \mathrm{Cd}, \mathrm{Be}$, and $\mathrm{Sr}$ [15], but the lack of associated $p$-type activation, in contrast to $\mathrm{Mg}$, has not been explained.

Adding small amounts of $\mathrm{Mg}$ to $\mathrm{GaN}$ leads to characteristic peaks in the UV range of the PL spectrum, at 3.27 and $3.466 \mathrm{eV}$ (measured at $T=2 \mathrm{~K}$ ) [16], and in some samples a peak at $\sim 3.2 \mathrm{eV}[17,18]$. The peak at $3.466 \mathrm{eV}$ is also observed in samples doped with other divalent cations [15] and is attributed to acceptor-bound excitons (ABEs) [19]. Interestingly, there is some evidence that this peak occurs in nominally undoped GaN samples [16], indicating that it may instead relate to some compensating native shallow defect. When the Mg content increases, the PL spectrum changes, becoming dominated by a broad peak at $\sim 2.9 \mathrm{eV}$, corresponding to blue luminescence (BL) and attributed to donor-acceptor pairs (DAPs) [16]. BL has also been observed in samples when $T$ is raised, rather than the $\mathrm{Mg}$ concentration ([Mg]) [17]. Thus far, theory fails to account for the characteristic UV luminescence associated with small $[\mathrm{Mg}]$, but recombination processes have been proposed to account 
for BL and also red luminescence [9-12], which is sometimes observed in heavily doped samples.

Recent thermal admittance spectroscopy measurements on epitaxially grown $n$-GaN have found that the shallow donor level lies $51 \mathrm{meV}$ below the conduction band minimum (CBM) [20], agreeing with older measurements using electron irradiation techniques [21] (placing the level at $64 \pm 10 \mathrm{meV}$ below the CBM). This level is significantly deeper than those introduced when $\mathrm{GaN}$ is intentionally doped $n$ type with, e.g., Si or O ( 30 meV) [15]. Such a shallow positive defect may act as a compensating center for holes in $\mathrm{GaN}$, though this has not conclusively been shown to be the case.

In this Letter, we present calculated formation and ionization energies of nitrogen vacancies $\left(V_{\mathrm{N}}\right)$ and $\mathrm{Mg}$ substituting on a Ga site $\left(\mathrm{Mg}_{\mathrm{Ga}}\right)$ in $\mathrm{GaN}$ in the dilute limit, using a multiscale embedded cluster approach. We also calculate the ionization energies associated with the divalent substitutionals: $\mathrm{Be}_{\mathrm{Ga}}, \mathrm{Zn}_{\mathrm{Ga}}, \mathrm{Cd}_{\mathrm{Ga}}$, and $\mathrm{Hg}_{\mathrm{Ga}}$ in the dilute limit. We find isolated $\mathrm{Mg}_{\mathrm{Ga}}$ do not contribute to $p$-type conductivity, instead they are compensated by $V_{\mathrm{N}}$ formation. The same result follows for the other divalent dopants considered. We find that the $V_{\mathrm{N}}$ is a shallow donor at $44 \mathrm{meV}$ below the CBM, accounting for the observed $3.466 \mathrm{eV}$ PL peak, and is stable in the negative charge state, facilitating Fermi level pinning close to (and above) the $\mathrm{CBM}$ and leading to native $n$-type conductivity. We determine that the equilibrium $\mathrm{Mg}_{\mathrm{Ga}}$ level lies $0.307 \mathrm{eV}$ above the VBM; we also calculate related levels that depend on the hole configuration and final spin state after excitation, that, although are slightly less favorable, will be accessible in fast PL experiments and account for the main peaks observed at low $T$ and $[\mathrm{Mg}]$. The observed BL is related to excitation and recombination of ionized $V_{\mathrm{N}}$, which will occur in greater concentrations at higher $T$ and $[\mathrm{Mg}]$, in agreement with experiment. We also find good agreement between our calculated ionization energies for $\mathrm{Be}, \mathrm{Zn}, \mathrm{Cd}$, and $\mathrm{Hg}$ impurities and relevant PL measurements. Our results give a simple but comprehensive explanation to the observed native $n$-type conductivity, PL spectrum at low Mg content, and the difficulty with $p$-type doping in GaN.

We employed the hybrid quantum mechanical (QM) and molecular mechanical (MM) embedded cluster method [22] to calculate bulk and defect energies in GaN. In this approach, a defect (possibly charged) and its immediately surrounding region, of the order of 100 atoms, is treated using a QM level of theory-here using density functional theory with a triple-zeta-plus-polarization Gaussian basis set (see Ref. [23] for details) and a hybrid exchange and correlation functional employing $42 \%$ exact exchange (BB1K) [24]. The QM region is embedded within a larger cluster, typically $10000-20000$ atoms, which is treated at a MM level of theory, using polarizable-shell interatomic potentials that accurately reproduce the dielectric, elastic, and structural properties of the bulk material [25]. Thus, one can model accurately isolated charged defects in any dimensionality [26], fully accounting for the dielectric response from the surrounding material. As supercells are not employed, there is no need to correct for imagecharge interactions. Crucially, ionization energies can be determined relative to a well-defined reference level, which we term the "vacuum level" (although in reality it is a "quasivacuum" level, because surface effects are not included in the energetics). Technical details, including the treatment of cluster termination and the interface between the QM and MM regions, are discussed elsewhere $[22,23]$. One caveat is that the defects modeled must be localized within the QM region, which, however, applies in the current case. This method was applied successfully to treat defects in $\mathrm{ZnO}$ [27] and band alignment in $\mathrm{TiO}_{2}$ [28].

We first consider the $\mathrm{Mg}_{\mathrm{Ga}}$ defect. We determine the formation energy of defect $X\left(E_{f}[X]\right)$ as

$$
E_{f}[X]=\Delta E(X)-\sum_{i} n_{i} \mu_{i}+q E_{F}
$$

where $\Delta E(X)$ is the difference in energy between the embedded cluster with and without $X, n_{i}$ is the number of atoms of species $i$ added $\left(n_{i}>0\right)$ or subtracted $\left(n_{i}<0\right)$ in forming $X, \mu_{i}$ is the chemical potential of species $i, q$ is the charge of $X$, and $E_{F}$ is the Fermi energy. For $q=0$, there are two localized hole configurations in the first coordination shell: either on a $\mathrm{N}$ in the basal plane of the surrounding tetrahedron of $\mathrm{N}$ atoms or on the axial $\mathrm{N}$ [for the related spin densities, see Figs. 1(a) and 1(b)]. Using Eq. (1), we find that, in anion-poor conditions (see Supplemental Material for details [29]), $E_{f}\left[\mathrm{Mg}_{\mathrm{Ga}}^{0}\right]$, with the hole localized on an axial $\mathrm{N}$, is $1.928 \mathrm{eV}$, in excellent agreement with previous calculations [11]. In anion-rich conditions, this energy changes to $0.783 \mathrm{eV}$. The axial hole energy is more favourable (by $0.063 \mathrm{eV}$ ) than that of the configuration with the hole localized on a $\mathrm{N}$ in the basal plane. If we consider $\mathrm{Mg}_{3} \mathrm{~N}_{2}$ as the source of $\mathrm{Mg}$, rather than Mg metal,

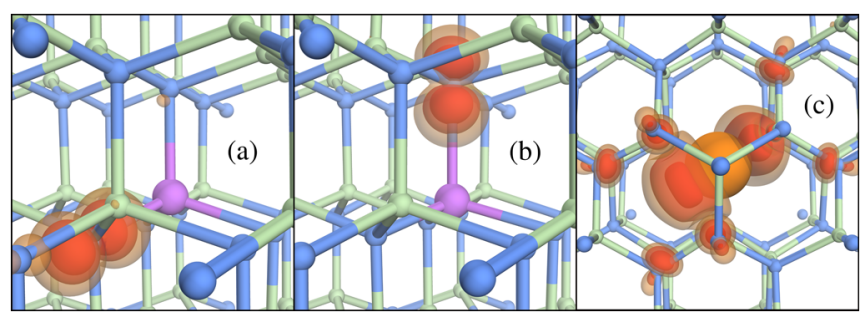

FIG. 1 (color online). Calculated spin density resulting from (a) a $\mathrm{Mg}_{\mathrm{Ga}}^{0}$-associated hole localized on a neighboring $\mathrm{N}$ in the basal plane, (b) a $\mathrm{Mg}_{\mathrm{Ga}}^{0}$-associated hole localized on a neighboring axial $\mathrm{N}$, and (c) a $\mathrm{N}$ vacancy. Light gray (green) [darker gray (blue)] spheres represent $\mathrm{Ga}(\mathrm{N})$ atoms. The darkest gray sphere represents a $\mathrm{Mg}$ atom in (a) and (b) (purple) and a vacancy in (c) (orange). Spin densities are indicated by (red) isosurfaces of density (au) 0.05, 0.025, and 0.01 for (a) and (b) and 0.01, 0.005, 0.0025 for $(\mathrm{c})$. 
we obtain a higher formation energy of $2.375 \mathrm{eV}(2.756 \mathrm{eV})$ for anion-rich (anion-poor) conditions.

The energy to dissociate a hole, according to the reaction

$$
\mathrm{Mg}_{\mathrm{Ga}}^{0} \rightarrow \mathrm{Mg}_{\mathrm{Ga}}^{-}+h^{+},
$$

is highly unfavorable at $1.404 \mathrm{eV}$. This result differs from the low value of $0.26 \mathrm{eV}$ obtained using periodic supercell models [11], which may suffer from incomplete cancellation of the electron self-interaction $[31,32]$ and treatment of long-range polarization, as well as an absence of a welldefined reference [33]. Our result, however, is consistent with earlier work on thermodynamical doping limits in $\mathrm{GaN}$ [23], which showed that free holes are unstable with respect to point defect formation (agreeing with the natively $n$-type nature of $\mathrm{GaN}$ ). Indeed, considering the compensation of holes by the formation of $V_{\mathrm{N}}$,

$$
h^{+}+\frac{1}{3} \mathrm{~N}_{\mathrm{N}}^{0} \leftrightarrow \frac{1}{3} V_{\mathrm{N}}^{3+}+\frac{1}{6} \mathrm{~N}_{2},
$$

we determine a reaction energy of $-1.245 \mathrm{eV}$; i.e., the balance is far to the right, indicating the thermodymical instability of holes. Consequently, doping with low levels of $\mathrm{Mg}$ will not result in free-hole formation, instead compensation by $V_{\mathrm{N}}$ will occur. We stress that our calculations apply to the dilute limit, under the assumption of thermodynamical equilibrium. For certain designs and/or synthetic procedures, where kinetic effects may dominate, or at higher $[\mathrm{Mg}]$, where the formation of complexes or phase segregated nanostructures may occur, the balance of the reaction [Eq. (3)] could shift to the left.

We show the formation energy of $V_{\mathrm{N}}$ (assuming $\mathrm{N}$-rich conditions), determined using Eq. (2), as a function of $E_{F}$ relative to the VBM in Fig. 2 [see Fig. 1(c) for the related spin density]. We find that, in $n$-type conditions, the $V_{\mathrm{N}}$ is singly ionized, indicating that it is shallow. In $p$-type conditions, the formation of $V_{\mathrm{N}}^{3+}$ becomes spontaneous, indicating the instability of free holes. $V_{\mathrm{N}}^{2+}$ is unfavorable at any value of $E_{F}$ within the gap. $V_{\mathrm{N}}^{+}$and $V_{\mathrm{N}}^{3+}$ are equally

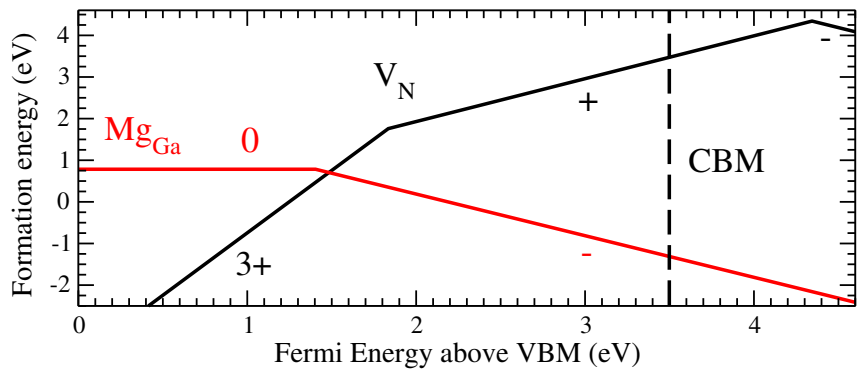

FIG. 2 (color online). Formation energy of $V_{\mathrm{N}}$ (black line) and $\mathrm{Mg}_{\mathrm{Ga}}$ [light gray (red) line] as a function of Fermi energy above the VBM. Anion-rich conditions are assumed. The position of the conduction band minimum (CBM) is indicated by the broken line. For each value of Fermi energy, only the most stable defect charge state is shown, with a change in slope indicating a change in charge state. favourable at $E_{F}=1.835 \mathrm{eV}$, a result significantly higher than those determined using plane-wave basis set calculations $[8,10]$ (see the above discussion on self-interaction errors). $V_{\mathrm{N}}^{-}$becomes favorable in the regime of degenerate $n$-type conduction at $\sim 0.9 \mathrm{eV}$ above the CBM, where it is expected to pin $E_{F}$. Integrating the density of states up to this $E_{F}$ gives an $n$-type concentration of $\sim 10^{20} \mathrm{~cm}^{-3}$, which has been observed in some undoped $n$-type samples [35].

In Fig. 3(a), we show the calculated vertical ionization energies (IEs) of $\mathrm{Mg}_{\mathrm{Ga}}$ relative to the conduction and valence bands. The IE is defined as the difference between the energy of a defect in charge state $q$ and the energy of the defect in the same configuration, but with charge $q+1$. The VBM relative to the vacuum level is determined by considering ionization of an electron from the bulk system. We include ionization of $\mathrm{Mg}_{\mathrm{Ga}}^{0}$ and $\mathrm{Mg}_{\mathrm{Ga}}^{+}$with both hole configurations (i.e., with the hole localized on a basal plane $\mathrm{N}$ or axial $\mathrm{N}$ ), as both should be accessible from electron excitation experiments such as PL. For the same reason, we include both possible final states, singlet and triplet, for the case of ionization of $\mathrm{Mg}_{\mathrm{Ga}}^{0}$. Such IEs correspond to emission

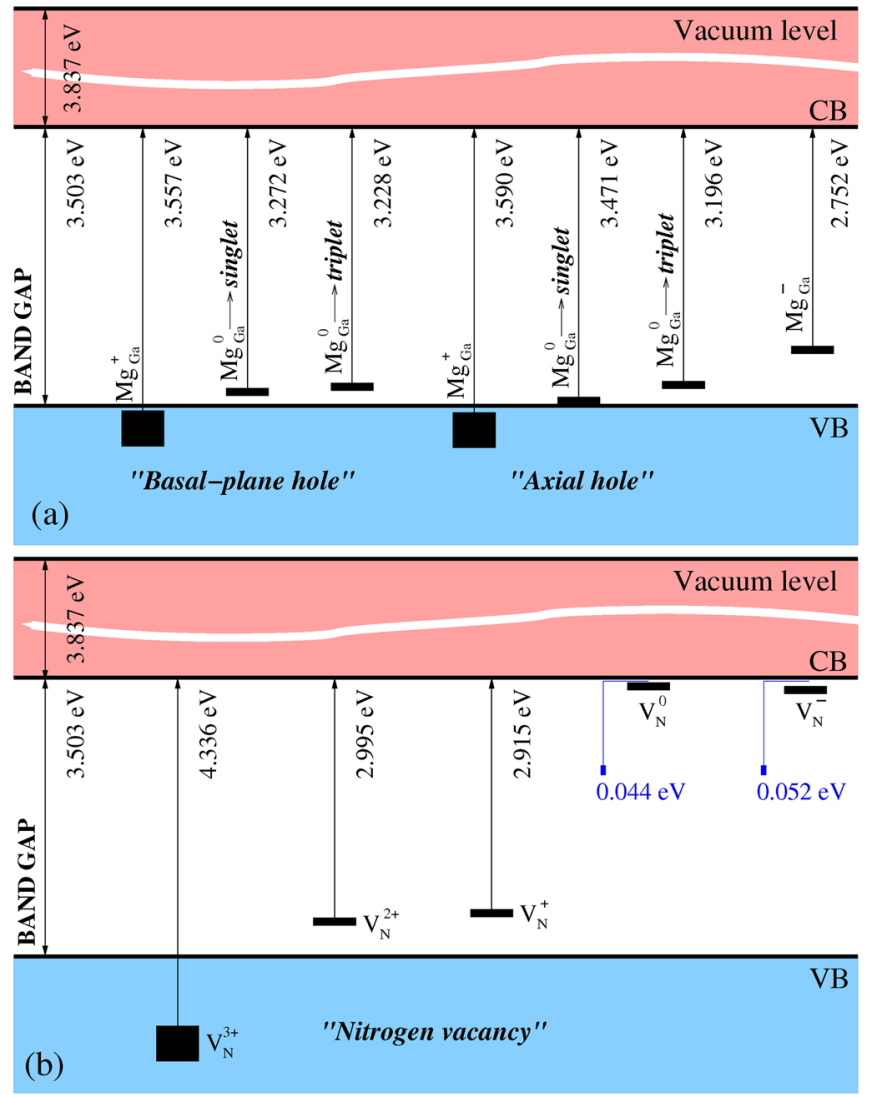

FIG. 3 (color online). Calculated vertical ionization levels of (a) $\mathrm{Mg}_{\mathrm{Ga}}$ and (b) $V_{\mathrm{N}}$ in the relevant charge states, shown relative to the valence band (VB), conduction band (CB), and vacuum level. In (a) the two hole configuration cases, consisting of localization on a $\mathrm{N}$ either in the basal plane or along the $c$ axis, are shown. For the neutral state, ionization to either singlet or triplet states is included. The large black boxes indicate resonance states in the VB. 
TABLE I. Calculated photoluminescence transitions, from total energy calculations, for different hole configurations and corresponding optical defect ionization levels compared with relevant experimental results taken from Refs. [15-17,37-43].

\begin{tabular}{|c|c|c|c|c|c|c|c|c|}
\hline \multirow[b]{3}{*}{ Final state } & \multicolumn{5}{|c|}{ Photoluminescence $(\mathrm{eV})$} & \multicolumn{3}{|c|}{ Levels (meV) } \\
\hline & \multicolumn{2}{|c|}{ Basal-plane hole } & \multicolumn{2}{|c|}{ Axial hole } & \multirow[t]{2}{*}{ Experiment } & & & \multirow[t]{2}{*}{ Experiment } \\
\hline & Singlet & Triplet & Singlet & Triplet & & 0 & $1-$ & \\
\hline $\mathrm{Be}$ & 3.410 & 3.427 & 3.454 & 3.456 & $3.384,3.466$ & $93,76,49,47$ & 624 & 90 \\
\hline $\mathrm{Mg}$ & 3.272 & 3.228 & 3.471 & 3.196 & $3.21,3.27,3.466$ & $275,231,307,32$ & 751 & 224,290 \\
\hline $\mathrm{Zn}$ & 3.144 & 3.201 & 3.068 & 3.195 & $3.100,3.45$ & $359,302,435,308$ & 975 & $340,400,480$ \\
\hline $\mathrm{Cd}$ & 2.845 & 2.929 & 2.814 & 2.934 & $2.8,2.937,3.455$ & $658,574,689,569$ & 1197 & 560 \\
\hline $\mathrm{Hg}$ & 2.584 & 2.666 & 2.587 & 2.694 & 2.70 & $919,837,916,809$ & 1455 & 410,800 \\
\hline
\end{tabular}

energies of photoexcited electrons recombining with these defect levels, as would be observed in PL experiments, where, after excitation, atoms typically do not have adequate time to fully relax. Our calculations are in excellent agreement with the low $T$ and $[\mathrm{Mg}] \mathrm{PL}$ spectra observed experimentally [16,17]. We reproduce well the DAP PL peaks at 3.21 and $3.27 \mathrm{eV},[36]$ and the ABE peak at $3.466 \mathrm{eV}$. The $2.75 \mathrm{eV} \mathrm{IE} \mathrm{of} \mathrm{Mg}_{\mathrm{Ga}}^{-}$, although in approximate agreement with the observed BL peak, is unlikely to be observed due to its high formation energy $(1.404 \mathrm{eV})$.

The vertical IEs of $V_{\mathrm{N}}$ are, similar to the case of $\mathrm{Mg}_{\mathrm{Ga}}$, shown in Fig. 3(b). We find that $V_{\mathrm{N}}^{0}$ is a shallow donor, having a vertical IE at $44 \mathrm{meV}$ below the CBM, in good agreement with measurements using thermal admittance spectroscopy and electron irradiation techniques [20,21]. This level may also account for the $3.46 \mathrm{eV}$ PL peak observed in a wide range of divalently doped and undoped GaN samples [15-17]. $V_{\mathrm{N}}^{-}$is also stable and shallow with a vertical IE $52 \mathrm{meV}$ below the CBM. The stability and near degeneracy of the $V_{\mathrm{N}}^{0}$ and $V_{\mathrm{N}}^{-}$states facilitates Fermi level pinning near the CBM. When the $V_{\mathrm{N}}$ donates electrons and relaxes to its equilibrium configurations, the resulting IEs are deep, and in excellent agreement with the $2.95 \mathrm{eV} \mathrm{BL}$ peak that is observed at higher $[\mathrm{Mg}]$ and at higher $T$ $[16,17]$. We associate these levels with the BL, as there will be more ionized $V_{\mathrm{N}}$ present at higher $T$ and also at higher $[\mathrm{Mg}]$ due to an increased number of compensating $V_{\mathrm{N}}$.

In Table I we present our calculated IEs associated with $B e_{\mathrm{Ga}}, \mathrm{Mg}_{\mathrm{Ga}}, \mathrm{Zn}_{\mathrm{Ga}}, \mathrm{Cd}_{\mathrm{Ga}}$, and $\mathrm{Hg}_{\mathrm{Ga}}$, along with the resulting defect levels, compared with relevant experiment. In each case the agreement is good. For all cases there is an observed $3.46 \mathrm{eV}$ peak, but only for the cases of $\mathrm{Be}_{\mathrm{Ga}}$ and $\mathrm{Mg}_{\mathrm{Ga}}$ can we attribute it to an $\mathrm{ABE}$. For the other dopants we attribute the peak to the compensating $V_{\mathrm{N}}$ (which may also play a role in $\mathrm{Be}$ and $\mathrm{Mg}$ doping).

We therefore arrive at a simple explanation for what occurs when $\mathrm{Mg}$ or other divalent metal dopants are added to $\mathrm{GaN}$ in small concentrations. Isolated $\mathrm{Mg}_{\mathrm{Ga}}$ strongly trap holes and, therefore, do not contribute to $p$-type conduction, instead hole carriers will be compensated by the formation of $V_{\mathrm{N}}$, a result that follows for other divalent metal dopants [44]. The $V_{\mathrm{N}}$ are shallow donors, with the near degeneracy of the neutral and negative charge states pinning the Fermi level close to the CBM, giving rise to the native $n$-type conductivity. Our calculated IEs are in excellent agreement with the relevant PL spectra. Furthermore, the $V_{\mathrm{N}}$ level can give rise to the $3.46 \mathrm{eV}$ peak observed in a wide range of doped and undoped samples, as it will be present as a compensating center (for the case of $\mathrm{Mg}$ and $\mathrm{Be}$ doping the $3.46 \mathrm{eV}$ peak can also be attributed to ABEs, which for $\mathrm{Mg}$ doping agrees with experiment $[13,15])$. A comprehensive description of PL and conductivity phenomena in $\mathrm{GaN}$ lightly doped with $\mathrm{Mg}, \mathrm{Be}, \mathrm{Zn}, \mathrm{Cd}$, and $\mathrm{Hg}$ is thus provided, without the need to propose, in the technologically significant case of $\mathrm{Mg}$ doping in particular, clustering of $\mathrm{Mg}_{\mathrm{Ga}}$ and $V_{\mathrm{N}}$, or $\mathrm{H}$ impurities. The latter may, however, play an important role in heavily doped $\mathrm{GaN}$, which is less well characterized or understood. The key feature of such a material is the presence of inverted Mg-rich pyramidal domains [45], which could trap interstitial $\mathrm{N}$, making vacancy formation unfavorable, and lead to lower hole ionization potentials. Such hypotheses, however, require appropriate investigation and are beyond the scope of this study.

In summary, we have comprehensively studied defect formation associated with divalent metal doping in $\mathrm{GaN}$, using a multiscale approach. Our results explain in detail the process by which low levels of divalent dopants are compensated by $V_{\mathrm{N}}$, and are in excellent agreement with available PL experimental data.

We acknowledge funding from EPSRC Grants No. EP/ D504872, No. EP/I01330X/1, and No. EP/K016288/1. M. M. acknowledges support from Accelerys Ltd. The authors also acknowledge the use of the UCL Legion High Performance Computing Facility and associated support services, the IRIDIS cluster provided by the EPSRC funded Centre for Innovation (EP/K000144/1 and EP/ K000136/1), and the ARCHER supercomputer through membership of the UK's HPC Materials Chemistry Consortium (EPSRC Grant No. EP/L000202). A. W. thanks C. G. Van de Walle (UCSB) for useful discussions. A.W. and D. O.S. acknowledge membership of the Materials Design Network. We would like to thank C. Humphreys, T. D. Veal, and K. P. O'Donnell for useful discussions. 
*j.buckeridge@ucl.ac.uk

a.sokol@ucl.ac.uk

[1] S. Nakamura and M. Krames, Proc. IEEE 101, 2211 (2013).

[2] S. Strite and H. Morkoç, J. Vac. Sci. Technol. B 10, 1237 (1992).

[3] H. Morkoç, Handbook of Nitride Semiconductors and Devices, (Wiley-VCH, Weinheim, 2008), Vol. 1.

[4] III-Nitride Based Light Emitting Diodes and Applications, edited by T.-Y. Seong, J. Han, H. Amano, H. Morkoc (Springer, Berlin, 2013).

[5] L. S. Chuah, Z. Hassan, S. S. Ng, and H. Abu Hassan, J. Mater. Res. 22, 2623 (2007).

[6] B. Monemar, Phys. Rev. B 10, 676 (1974).

[7] O. Madelung, Semiconductors: Data Handbook, 3rd ed. (Springer, Berlin, 2004).

[8] C. G. V. de Walle and J. Neugebauer, J. Appl. Phys. 95, 3851 (2004).

[9] S. Lany and A. Zunger, Appl. Phys. Lett. 96, 142114 (2010).

[10] Q. Yan, A. Janotti, M. Scheffler, and C. G. Van de Walle, Appl. Phys. Lett. 100, 142110 (2012).

[11] J. L. Lyons, A. Janotti, and C. G. Van de Walle, Phys. Rev. Lett. 108, 156403 (2012).

[12] D. Lee, B. Mitchell, Y. Fujiwara, and V. Dierolf, Phys. Rev. Lett. 112, 205501 (2014).

[13] E. R. Glaser, W. E. Carlos, G. C. B. Braga, J. A. Freitas, Jr., W. J. Moore, B. V. Shanabrook, R. L. Henry, A. E. Wickenden, D. D. Koleske, H. Obloh, P. Kozodoy, S. P. DenBaars, and U. K. Mishra, Phys. Rev. B 65, 085312 (2002).

[14] M. E. Zvanut, Y. Uprety, J. Dashdorj, M. Moseley, and W. Alan Doolittle, J. Appl. Phys. 110, 044508 (2011).

[15] M. A. Reshchikov and H. Morkoç, J. Appl. Phys. 97, 061301 (2005).

[16] B. Monemar, P. P. Paskov, G. Pozina, C. Hemmingsson, J. P. Bergman, S. Khromov, V. N. Izyumskaya, V. Avrutin, X. Li, H. Morkoç, H. Amano et al., J. Appl. Phys. 115, 053507 (2014).

[17] M. Smith, G. D. Chen, J. Y. Lin, H. X. Jiang, A. Salvador, B. N. Sverdlov, A. Botchkarev, H. Morkoç, and B. Goldenberg, Appl. Phys. Lett. 68, 1883 (1996).

[18] B. Monemar, P. P. Paskov, G. Pozina, C. Hemmingsson, J. P. Bergman, T. Kawashima, H. Amano, I. Akasaki, T. Paskova, S. Figge et al., Phys. Rev. Lett. 102, 235501 (2009).

[19] R. Stępniewski, A. Wysmołek, M. Potemski, K. Pakuła, J. M. Baranowski, I. Grzegory, S. Porowski, G. Martinez, and P. Wyder, Phys. Rev. Lett. 91, 226404 (2003).

[20] A. O. Evwaraye, S. R. Smith, and S. Elhamri, J. Appl. Phys. 115, 033706 (2014).

[21] D. C. Look, D. C. Reynolds, J. W. Hemsky, J. R. Sizelove, R. L. Jones, and R. J. Molnar, Phys. Rev. Lett. 79, 2273 (1997).

[22] A. A. Sokol, S. T. Bromley, S. A. French, C. R. A. Catlow, and P. Sherwood, Int. J. Quantum Chem. 99, 695 (2004).

[23] A. Walsh, J. Buckeridge, C. R. A. Catlow, A. J. Jackson, T. W. Keal, M. Miskufova, P. Sherwood, S. A. Shevlin, M. B. Watkins, S. M. Woodley, and A. A. Sokol, Chem. Mater. 25, 2924 (2013).

[24] Y. Zhao, B. J. Lynch, and D. G. Truhlar, J. Phys. Chem. A 108, 2715 (2004).

[25] C. R. A. Catlow, Z. X. Guo, M. Miskufova, S. A. Shevlin, A. G. H. Smith, A. A. Sokol, A. Walsh, D. J. Wilson, and S. M. Woodley, Phil. Trans. R. Soc. A 368, 3379 (2010).
[26] J. Buckeridge, S. T. Bromley, A. Walsh, S. M. Woodley, C. R. A. Catlow, and A. A. Sokol, J. Chem. Phys. 139, 124101 (2013).

[27] A. A. Sokol, S. A. French, S. T. Bromley, C. R. A. Catlow, H. J. J. van Dam, and P. Sherwood, Faraday Discuss. 134, 267 (2007).

[28] D. O. Scanlon, C. W. Dunnill, J. Buckeridge, S. A. Shevlin, A. J. Logsdail, S. M. Woodley, C. R. A. Catlow, M. J. Powell, R. G. Palgrave et al., Nat. Mater. 12, 798 (2013).

[29] See Supplemental Material at http://link.aps.org/ supplemental/10.1103/PhysRevLett.114.016405, which includes Ref. [30], for a discussion of the defect reactions that correspond to anion-rich and anion-poor growth conditions.

[30] F. A. Kröger, The Chemistry of Imperfect Crystals (North-Holland, Amsterdam, 1974).

[31] G. Pacchioni, F. Frigoli, D. Ricci, and J. A. Weil, Phys. Rev. B 63, 054102 (2000).

[32] If we take into consideration the self-trapping energy of a hole in $\mathrm{GaN}$ at $0.3 \mathrm{eV}$, our result is in reasonable agreement with that of Lany and Zunger, who used a Koopmancorrected approach to treat this error.

[33] Indeed, we find a value of $0.863 \mathrm{eV}$ for the reaction energy, lower than $1.404 \mathrm{eV}$ but still above $0.26 \mathrm{eV}$, when using the B97-2 functional [34], which uses $21 \%$ exact exchange, an amount even lower than that used in Ref. [11]. Accurate formation energies require an accurate thermochemical method, which reproduces structure, atomization energies, and ionization potentials.

[34] P. J. Wilson, T. J. Bradley, and D. J. Tozer, J. Chem. Phys. 115, 9233 (2001).

[35] III-Nitride Semiconductors: Electrical, Structural and Defect Properties, edited by O. Manasreh (Elsevier Science, New York, 2000).

[36] Here we are assuming a donor level of 30-50 meV below the CBM, but excluding the Coulomb attraction energy between the donor and acceptor pair, as simply subtracting the donor level from the acceptor level values is equivalent to assuming infinite separation.

[37] M. A. L. Johnson, Z. Yu, C. Boney, W. C. Hughes, J. W. Cook, Jr., J. F. Schetzina, H. Zhao, B. J. Skromme, and J. A. Edmond, Mater. Res. Soc. Symp. Proc. 449, 215 (1996).

[38] E. Ejder and H. G. Grimmeiss, Appl. Phys. 5, 275 (1974).

[39] B. Monemar, O. Lagerstedt, and H. P. Gislason, J. Appl. Phys. 51, 625 (1980).

[40] B. Monemar, H. P. Gislason, and O. Lagerstedt, J. Appl. Phys. 51, 640 (1980).

[41] M. Ilegems, R. Dingle, and R. A. Logan, J. Appl. Phys. 43, 3797 (1972).

[42] J. I. Pankove and J. A. Hutchby, J. Appl. Phys. 47, 5387 (1976).

[43] F. J. Sánchez, F. Calle, M. A. Sánchez-García, E. Calleja, E. Muñoz, C. H. Molloy, D. J. Somerford, J. J. Serrano, and J. M. Blanco, Semicond. Sci. Technol. 13, 1130 (1998).

[44] As we showed previously [23], the high- $T$ treatment will not result in a significant shift in the equilibrium between the electronic and ionic defects in this and isostructural widegap semiconductors.

[45] P. Vennéguès, M. Benaissa, B. Beaumont, E. Feltin, P. De Mierry, S. Dalmasso, M. Leroux, and P. Gibart, Appl. Phys. Lett. 77, 880 (2000). 